\title{
Angular correlation of positron annihilation radiation in perfluorohexane mixtures with nonpolar liquids
}

\author{
O.E. MOGENSEN
}

Chemistry Department, Japan Atomic Energy Research Institute, Tokai-mura, Ibaraki-ken 319-11, Japan

non-refereed paper

\begin{abstract}
Positron-annihilation linear-slit angular-correlation (AC) spectra in perfluorohexane $\left(\mathrm{C}_{6} \mathrm{~F}_{14}\right)$ mixtures with hexane and isooctane in the entire concentration interval are reported. Annihilation with electrons in fluorine results in an unusually broad $\mathrm{AC}$-component. The data show that the free positron reacts to form a bound state with $\mathrm{C}_{6} \mathrm{~F}_{14}$ at low $\mathrm{C}_{6} \mathrm{~F}_{14}$ concentration. At high $\mathrm{C}_{6} \mathrm{~F}_{14}$ concentration the positron in Ps annihilates preferentially with electrons in the hydrocarbons, and not with the electrons in $\mathrm{C}_{6} \mathrm{~F}_{14}$ before this cannot be avoided. Hence, the energy of the Ps bubble state is lower if the surface consists of hydrocarbon molecules. While the positron in Ps penetrates further into the hydrocarbons than the electron in Ps, the two particles have probably about equal penetration in $\mathrm{C}_{6} \mathrm{~F}_{14}$, a weak electron acceptor. This difference causes probably a lower dispersion/polarization attraction energy of the molecules and Ps in $\mathrm{C}_{6} \mathrm{~F}_{14}$. It is conjectured that the Ps relative contact density increases from about 0.8 in hydrocarbons to roughly $1.0-1.1$ in weak electron acceptor liquids (e.g., $\mathrm{C}_{6} \mathrm{~F}_{14}$ ), and then decreases to a low value, i.e., $0.1-0.2$, at higher electron affinity.
\end{abstract}

\section{Introduction}

A fast positron entering condensed matter is nearly always thermalized before annihilating with an electron into photons. By studying the properties of the annihilation photons information on the various processes preceding the annihilation can be obtained. Positronium (Ps), the bound state of a positron and an electron, is formed in many insulating liquids and solids, but not in metals and semiconductors. One important criterion for Ps formation in molecular substances is that Ps is the lowest energy state for the electron-positron pair. Hence, Ps is formed only if the molecules have no or low electron and/or positron affinities [1-4].

Positronium is formed by a reaction between the thermalized positron and one of the thermalized electrons released by ionization of the molecules during the slowing down processes of the positron in the positron spur, which is the terminal cluster of radiation created intermediates in the last part of the positron track. Positronium formation competes with the other processes, such as electron positive-ion recombination and particle outdiffusion, in the positron spur [1-4]. Consequently, Ps formation investigations can give valuable new information on excess electron and positive ion spur processes of importance in radiation chemistry. In 
general, experimental and theoretical studies of track and spur processes are important parts of radiation physics and chemistry (e.g. $[5,6])$.

Another general aspect of positron annihilation research is the fact that the electron, positron, and Ps are the only light particles participating in low energy physics and chemistry, apart from their bound states such as, for example, Ps- Because of the small masses quantum mechanical effects are of great importance in the description of the states and chemical reactions of the light particles. In general, the behaviour of the excess electron in insulating liquids seems to be strongly correlated to that of the positron. However, Ps is normally in a bubble state in liquids, which is found for the excess electron only in liquid helium, neon, and hydrogen. Of course, a unique chemical reaction of the light particles is that involving two light particles, namely the Ps formation reaction in the positron spur [1-6].

The measurements discussed in this paper were made to study two effects. The first one concerns the effect of adding electron scavengers on the Ps yield in nonpolar liquids. Roughly speaking the scavengers forming stable anions on the reaction with the excess electron influenced the Ps yield in two different ways. For example, $\mathrm{CS}_{2}$ [7], $\mathrm{SF}_{6}[7], \mathrm{CH}_{3} \mathrm{Cl}$ [8], and perfluorohexane $\left(\mathrm{C}_{6} \mathrm{~F}_{14}\right)$ [9] decreased, while perfluorobenzene [10] $\left(\mathrm{C}_{6} \mathrm{~F}_{6}\right)$ and naphthalene [7] increased, the PS formation if added to normal, nompolar liquids at low concentrations. This could not be explained in terms of only the electron affinities of the scavengers. An improved spur model of Ps formation was then proposed [8]. It was realized that scavenging of the positron had to be taken into account in a more detailed way. The new assumptions were that the Ps yield decreases only if the added scavenger traps both the electron and the positron, while it increases if only one of the particles is trapped. In the latter case the trapped particle can be picked off by the other fast moving particle to form Ps if the trap is not too deep. If both particles are trapped, and hence, slowly moving, Ps formation cannot occur before the positron decays with a lifetime of about 400 ps for many electron-positron pairs. The proposal is further discussed in sect. 4 .

According to this explanation both particles are trapped on $\mathrm{C}_{6} \mathrm{~F}_{14}$, while $\mathrm{C}_{6} \mathrm{~F}_{6}$ traps only the electron. Angular correlation spectra are "fingerprints" of the positron state at annihilation, while the positron lifetimes in different states have almost similar values. Furthermore, positrons annihilating with electrons in fluorine give a particularly broad AC spectra [4,9], which is easy to detect. Hence, it was decided to compare AC spectra for solutions of hexane and isooctane with $\mathrm{C}_{6} \mathrm{~F}_{6}$ and $\mathrm{C}_{6} \mathrm{~F}_{14}$ to test the predictions.

The second reason for this work was simply to measure the rate of reaction of the free positron (i.e., not bound in Ps) with $\mathrm{C}_{6} \mathrm{~F}_{14}$ in the two solvents. Solvated positron reactions with various ions $\left(\mathrm{Cl}^{-} \mathrm{Br}^{-\prime} \mathrm{I}^{-} \mathrm{CN}^{-}\right.$and $\mathrm{SCN}^{-1}$ in polar solvents, mainly water, have been studied before [11]. However, positron reactions with molecules in nonpolar solvents have been studied very little. Compared with the situation for the other two light particles very little information on the positron states and reactions in liquids has been obtained, as yet.

The expected effects were found. A broadening of the AC spectra occurred on the addition of $\mathrm{C}_{6} \mathrm{~F}_{14}$ at low concentration. However, unexpectedly the strongest broadening was obtained on the addition of the last $5 \%$ of $\mathrm{C}_{6} \mathrm{~F}_{14}$ to the two solvents. The results presented here have been discussed very briefly in a section of a conference contribution [12].

The experimental measurements and data analyses are described in sect. 2 , followed by a description of the obtained results in sect. 3. Section 4 gives a detailed discussion and interpretation of the data in the context of what is known today about the behaviour of the positron and Ps in liquids. The conclusion is given in sect. 5. 


\section{Experimental and data analysis}

In the two-gamma annihilation process the two photons are emitted in opposite directions, except for a small deviation which is mainly determined by the finite momentum of the electronpositron pair at the time of the annihilation. A certain state of the positron-electron system gives a certain AC spectrum. Hence, by measuring the AC spectrum one can determine where the positron was situated at the time of the annihilation provided the AC spectra of the positron states in the solution are sufficiently different from each other.

The AC measurements were performed at Risø, Denmark. We used a conventional linear-slit angular-correlation apparatus, as previously described in $[9,11]$. The full width at half maximum ( $\mathrm{fwhm}$ ) of the angular resolution was roughly $1.15 \mathrm{mrad}$, of which $0.96 \mathrm{mrad}$ was caused by the finite slit widths. The solutions were contained in a glass cell covered with a polymer foil, through which the positrons from an external source of about $1.1 \times 10^{9} \mathrm{~Bq}(30 \mathrm{mCi}){ }^{22} \mathrm{NaCl}$ entered the cell.

The positron lifetimes (LT) were measured by determining the time interval between the detection of a $1.28 \mathrm{MeV}$ photon, emitted simultaneously with the positron from a ${ }^{22} \mathrm{Na}$ source, and the detection of one of the annihilation photons of $0.511 \mathrm{MeV}$. We used a standard fast-fast lifetime setup with a resolution of about fwhm $=400 \mathrm{ps}$. The shape of the resolution curve, used in the POSITRONFIT $[13,14]$ analysis, was fitted by three gaussians by use of the RESOLUTION $[13,14]$ program. The positron source consisted of ${ }^{22} \mathrm{NaCl}$ between two $7.5 \mu \mathrm{m}$ Kapton foils. The lifetime spectrum of the positrons annihilating in the foils was subtracted from the measured spectra before the final analysis.

All the angular correlation and lifetime samples were degassed by the freeze-thaw method.

At first, the AC spectra were corrected for a very small random background. They were then analyzed by use of the PAACFIT program [13,14]. This program fits an AC spectrum by a sum of gaussians, the fitting parameters being the intensities (i.e., the relative areas in \%) and the fwhm's of the gaussians, and the symmetry angle of the spectra. The program finds also the variances of the fit, $\Phi$, which are calculated on the assumption that the model is ideal, in which case $\Phi=1 \pm 0.2$. As our model was not ideal we had to accept bigger $\Phi$ - values. The program has been used with good results in many studies (see e.g. $[4,9,11,13,14]$ ).

All the measured spectra could be well fitted by use of three gaussians, of which the narrowest one was assumed to be caused by para-Ps intrinsic annihilation. A small computer program was used for the calculation of the H-parameters of the broad components of the AC curves given by the sum of the two broadest gaussians.

The LT-spectra could be well fitted by a sum of three exponentially decaying terms by use of POSITRONFIT [13,14]. As the LT-spectra were only of standard quality, the "fourth" LTcomponent due to secondary Ps reactions with mainly positive ions $[15,16]$ could not be resolved. Hence, this process was disregarded in the analyses of the AC-and LT-spectra.

\section{Results}

We measured the AC-spectrum for about 42 different mixtures of perfluorohexane $\left(\mathrm{C}_{6} \mathrm{~F}_{14}\right)$ with n-hexane and 2,2,4-trimethylpentane (isooctane). The results were compared with positron LTresults for the same mixtures measured by Wikander [10]. At Risø we measured the following LT-results for $\mathrm{C}_{6} \mathrm{~F}_{14}$ / hexane mixtures: $0.01 \mathrm{M}, 3.82 \mathrm{~ns}, 38.8 \% ; 0.4 \mathrm{M}, 3.75 \mathrm{~ns}, 28.7 \% ; 2.0 \mathrm{M}, 4.30$ ns, $31.0 \%$; $4.0 \mathrm{M}, 4.89 \mathrm{~ns}, 23.8 \%$; pure $\mathrm{C}_{6} \mathrm{~F}_{14}(4.98 \mathrm{M}), 4.69 \mathrm{~ns}, 36.2 \%$, for the concentration, long 
Table 1. Fitting parameters for some angular correlation spectra. $I_{i}\left(f_{w h m}\right)$ is the relative intensity (full width at half maximum) of the $i^{\prime}$ th gaussian in a fit of a sum of three gaussians to the spectra

\begin{tabular}{|c|c|c|c|c|c|c|c|}
\hline Fitting curve for & $\begin{array}{l}\mathrm{I}_{1} \\
(\%)\end{array}$ & $\begin{array}{l}\text { fwhm }_{1} \\
\text { (mrad) }\end{array}$ & $\begin{array}{l}I_{2} \\
(\%)\end{array}$ & $\begin{array}{l}\text { fwhm }_{2} \\
\text { (mrad) }\end{array}$ & $\begin{array}{l}I_{3} \\
(\%)\end{array}$ & $\begin{array}{l}\text { fwhm }_{3} \\
\text { (mrad) }\end{array}$ & variance \\
\hline n-hexane & 16.66 & 2.54 & 80.88 & 8.57 & 2.45 & 20.34 & 3.64 \\
\hline isooctane & 17.47 & 2.68 & 79.64 & 8.54 & 2.89 & 17.83 & 1.78 \\
\hline $\mathrm{C}_{6} \mathrm{~F}_{14}(4.98 \mathrm{M})$ & 17.57 & 2.44 & 63.47 & 10.39 & 18.95 & 17.15 & 1.41 \\
\hline $\mathrm{C}_{6} \mathrm{~F}_{6}(8.66 \mathrm{M})$ & 17.42 & 2.66 & 67.76 & 10.21 & 14.83 & 17.54 & 2.17 \\
\hline $0.27 \mathrm{M} \mathrm{C}_{6} \mathrm{~F}_{14} /$ hex. & 11.00 & 2.45 & 83.01 & 9.00 & 5.99 & 19.62 & 3.98 \\
\hline 2.94 $\mathrm{M} \mathrm{C}_{6} \mathrm{~F}_{14} /$ hex. & 10.81 & 2.30 & 81.90 & 9.20 & 7.30 & 20.00 & 3.45 \\
\hline $4.48 \mathrm{M} \mathrm{C}_{6} \mathrm{~F}_{14} /$ hex. & 9.83 & 2.30 & 81.18 & 9.42 & 8.99 & 18.91 & 2.55 \\
\hline $0.24 \mathrm{M} \mathrm{C}_{6} \mathrm{~F}_{6} /$ hex. & 17.10 & 2.58 & 80.76 & 8.66 & 2.14 & 20.56 & 1.71 \\
\hline 4.63 $\mathrm{M} \mathrm{C}_{6} \mathrm{~F}_{6} /$ hex. & 17.76 & 2.54 & 74.49 & 9.14 & 7.75 & 17.83 & 1.10 \\
\hline 7.46 $\mathrm{M} \mathrm{C}_{6} \mathrm{~F}_{6} /$ hex. & 14.79 & 2.54 & 74.20 & 9.60 & 11.01 & 18.04 & 1.38 \\
\hline 8.39 $\mathrm{M} \mathrm{C}_{6} \mathrm{~F}_{6} /$ hex. & 16.36 & 2.64 & 71.70 & 10.14 & 11.95 & 18.61 & 1.51 \\
\hline
\end{tabular}

lifetime, and its intensity, respectively. We used a fixed short lifetime equal to $125 \mathrm{ps}$ in the analyses. These results are roughly equal to those reported in [10]. Furthermore, the results are compared with $A C$ - and LT-results for hexafluorobenzene $\left(\mathrm{C}_{6} \mathrm{~F}_{6}\right)$ mixtures with n-hexane, published previously [9].

The fitting parameters for the three-gaussian fits of some of the AC-spectra are shown in table 1. In figs. 1 and 2 the intensity of the narrowest gaussian in the free three-gaussian fits is plotted for $\mathrm{C}_{6} \mathrm{~F}_{14}$ mixtures with hexane and isooctane, respectively. Furthermore, we have plotted a third of the intensity of the longest lifetime in the fits of the LT-spectra by a sum of three exponentially decaying term, as extracted from the data reported by Wikander [10]. Clearly, the apparent para-Ps yield determined from the AC-spectra is larger than a third of the apparent ortho-Ps yield determined from the LT-spectra, if the simplest possible interpretation is used. A more detailed model will be discussed in sect. 4 . Nevertheless, the Ps yields extracted in a simple way from the AC-spectra are qualitatively very similar to those similarly extracted from the LT-spectra. In addition, it can be seen in fig. 2 that the inhibition of the Ps formation at low concentration is greater in isooctane than in hexane.

However, the most important results reported in this work are the shapes of the broad ACcomponents due to free positron and Ps pick-off annihilations. The broad AC-components, obtained by a subtraction of the narrowest gaussian in the free three-gaussian fits from the measured spectra, are plotted in fig. 3 for hexane, $C_{6} F_{14}, 0.27 C_{6} F_{14} /$ hexane, and $4.48 \mathrm{M}$ $\mathrm{C}_{6} \mathrm{~F}_{14}$ /hexane. Similar results were measured for the isooctane mixtures. Obviously, profound changes in the shape of the broad AC-components were observed on changing the $\mathrm{C}_{6} \mathrm{~F}_{14}$ concentrations.

We decided to illustrate these results by plotting the H-parameter for the broad AC-components versus $\mathrm{C}_{6} \mathrm{~F}_{14}$ concentration for hexane and isooctane mixtures in figs. 4 and 5, respectively. The plotted H-parameter is defined as the counts in top of the broad components divided by the areas of the broad components between $-15 \mathrm{mrad}$ and $+15 \mathrm{mrad}$., the broad components being defined as the sum of the two broadest gaussians in the free three-gaussian fits. The $\mathrm{H}-$ parameter, defined in the same way, is also plotted for mixtures of $\mathrm{C}_{6} \mathrm{~F}_{6}$ with hexane in figs. 4 


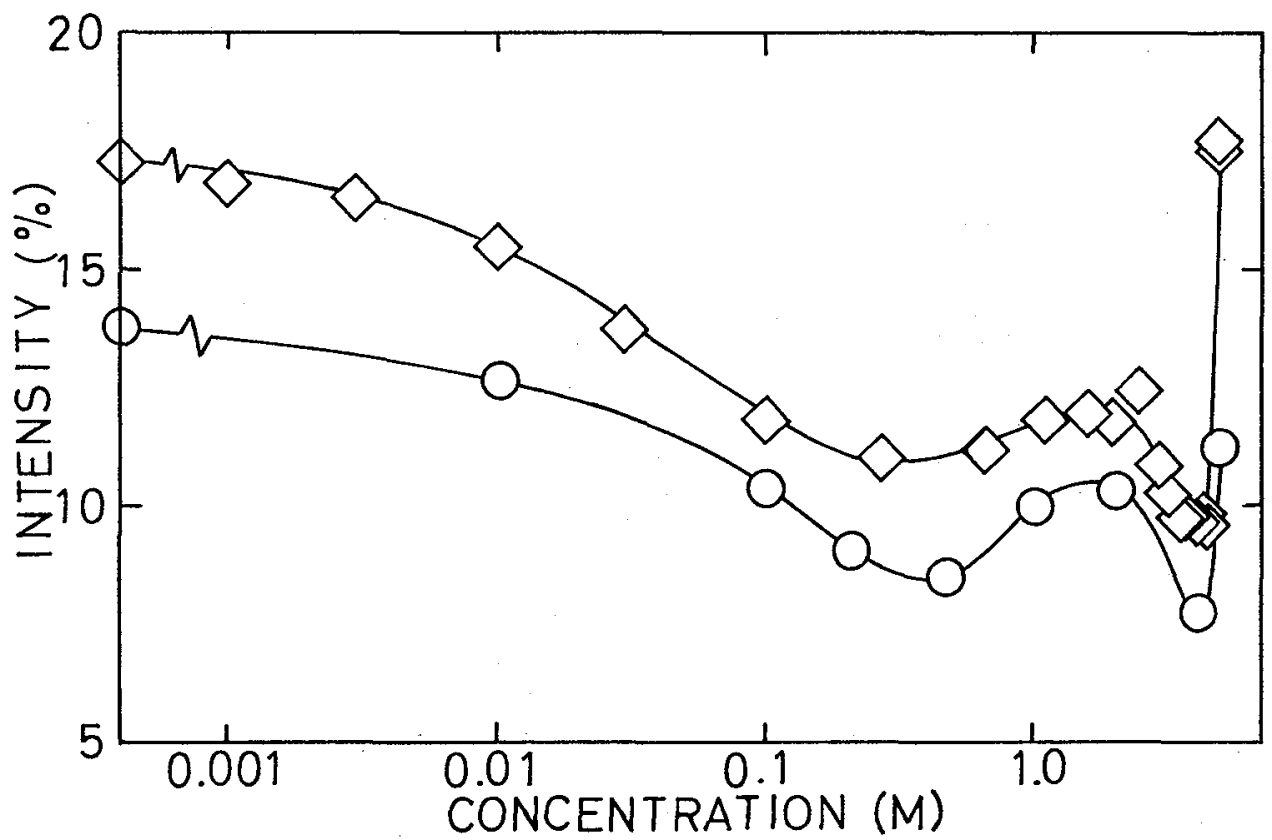

Fig. 1. Comparison of the Ps yields as determined by analyses of the AC-and LT-spectra for $\mathrm{C}_{6} \mathrm{~F}_{14}$ mixtures with hexane. OOOOOO : a third of the intensity of the longest LT-component [10]. $\infty>0-\infty)$ : the intensity of the narrowest gaussians in the AC-spectra. Lines are for guidance only.

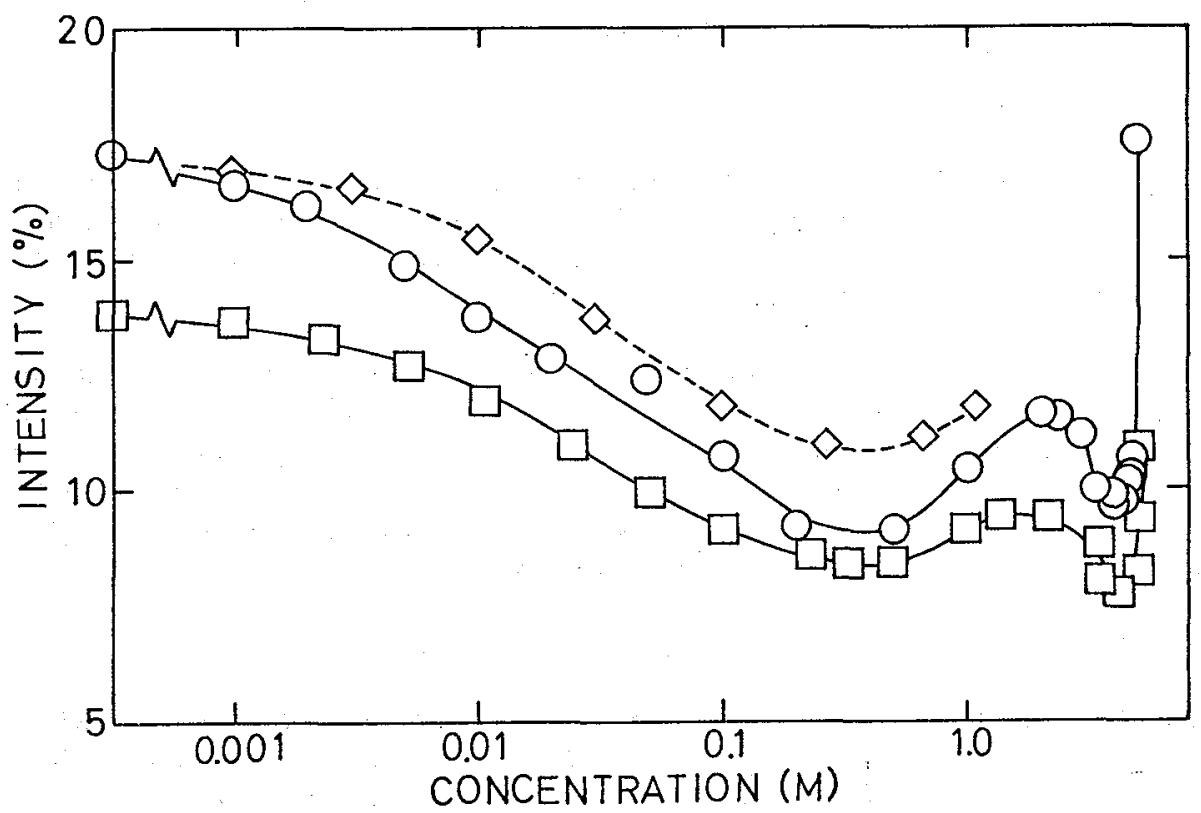

Fig. 2. Comparison of the Ps yields as determined by analyses of the AC- and LT-spectra for $\mathrm{C}_{6} \mathrm{~F}_{14}$ mixtures with isooctane. $\square \square \square \square \square \square$ : a third of the intensity of the longest LT-component [10]. 000000 : the intensity of the narrowest gaussians in the AC-spectra. $-\infty \Delta-\infty)$ : the intensity of the narrowest gaussians for the hexane mixtures. Lines are for guidance only. 


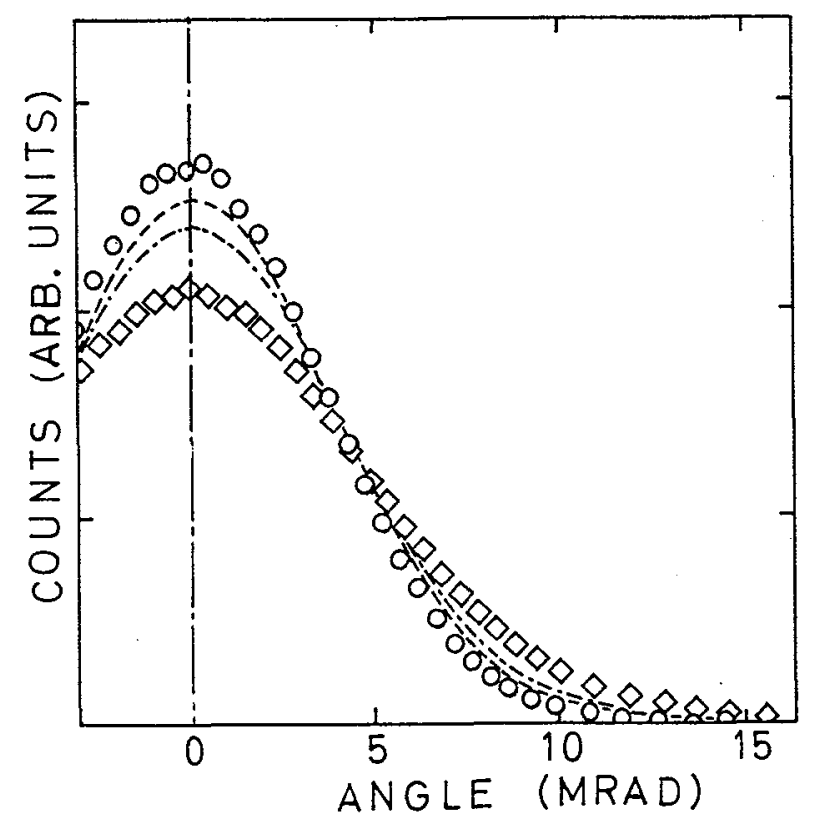

Fig. 3. The broad AC-components, given by the measured spectra minus the narrowest gaussian, for hexane (OOOO), $\mathrm{C}_{6} \mathrm{~F}_{14}(4.98 \mathrm{M})$ $(\infty \infty \infty), 0.27 \mathrm{M} \mathrm{C}_{6} \mathrm{~F}_{14}$ /hexane (- - -), and $4.48 \mathrm{M} \mathrm{C}_{6} \mathrm{~F}_{14} /$ hexane $(\cdot \cdots-)$. The spectra are normalized to the same area.

and 5. The narrow $\mathrm{AC}$-component and the long lifetime intensities for the $\mathrm{C}_{6} \mathrm{~F}_{6} /$ hexane mixtures have previously been reported in fig. 5 of [9]. The advantage of plotting the H-parameter is that it is linearly dependent on the yield of free positron and pick-off annihilations. For example, the fwhm does not fulfill that criteria.

As seen in figs. 4 and 5, the H-parameter versus $\mathrm{C}_{6} \mathrm{~F}_{14}$ concentration is very similar for the hexane and isooctane mixtures. A strong decrease in $\mathrm{H}$ is observed at low concentrations. However, a still stronger decrease occurs on adding the last percentage of $\mathrm{C}_{6} \mathrm{~F}_{14}$ to the mixtures. On the other hand, the $\mathrm{H}$-parameter depends almost linearly on the $\mathrm{C}_{6} \mathrm{~F}_{6}$ concentration in $\mathrm{C}_{6} \mathrm{~F}_{6}$ / hexane mixtures. Hence, the $\mathrm{H}$-parameter results for the $\mathrm{C}_{6} \mathrm{~F}_{14}$ mixtures are qualitatively very different from those of the $\mathrm{C}_{6} \mathrm{~F}_{6}$ / hexane mixtures.

\section{Discussion}

To facilitate the understanding of the interpretation of the measured results a brief discussion of the information obtained by the experimental methods, of the basic principles of the positron and Ps behaviour, and of Ps formation in condensed matter is given [3,4].

High quality LT-spectra for samples in which Ps is formed consist of four components, namely the para-Ps, free positron, ortho-Ps (and para-Ps) pick-off, and the socalled "fourth" components. Ortho-Ps (parallel spins) in condensed matter decays mainly into two photons by pick-off 


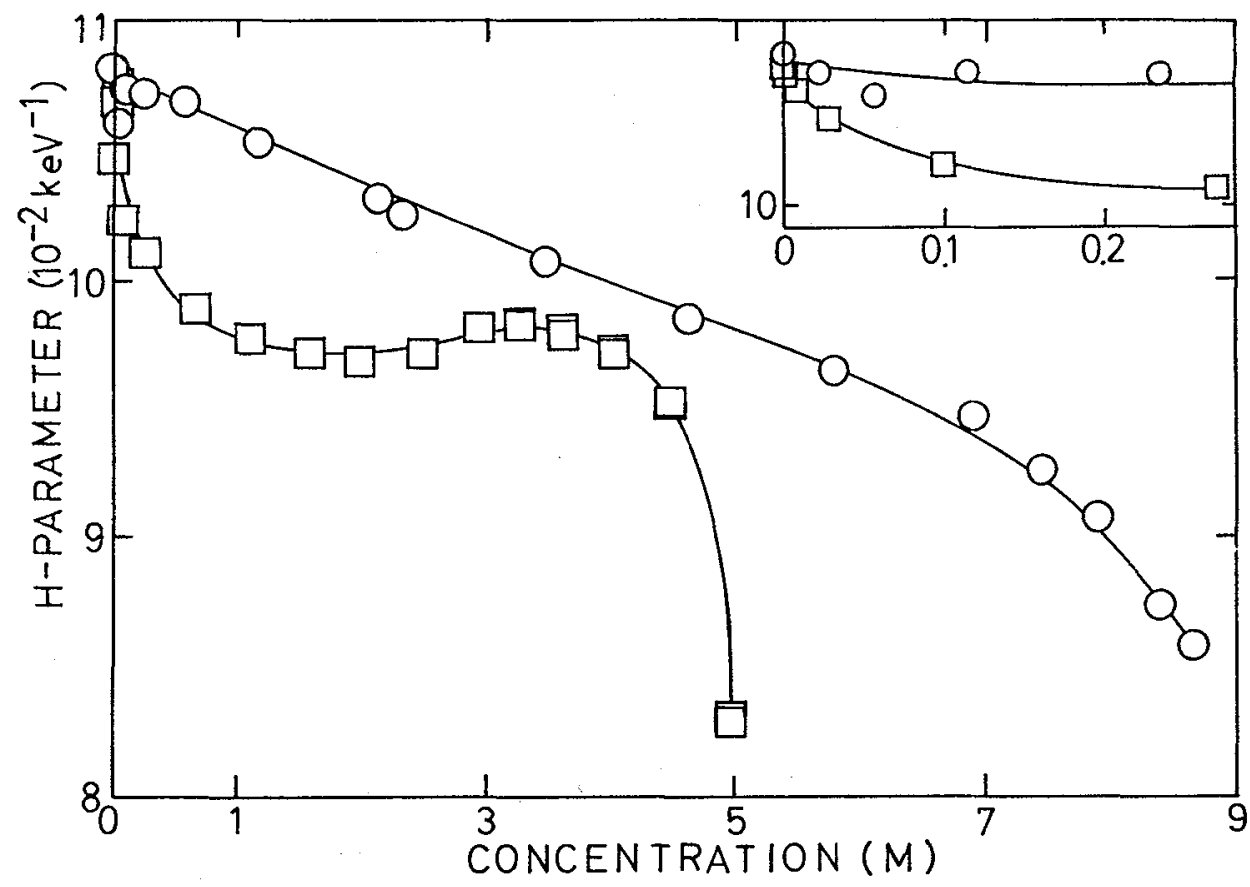

Fig. 4. H-parameter for the broad $A C$-component versus $C_{6} F_{14}(\square \square \square \square)$ and $C_{6} F_{6}(O O O O)$ concentration in hexane mixtures. The insert shows the low concentration results. Lines are for guidance only.

annihilation (lifetime $\tau=1-10 \mathrm{~ns}$ ), in which the positron in Ps annihilates with an electron having opposite spin direction during collisions with the molecules. The intrinsic three-photon annihilation of ortho-Ps (found in vacuum) is too slow ( $\tau=139 \mathrm{~ns}$ ) to play a role. Para-Ps (opposite spin directions) annihilates intrinsically into two photons ( $\tau=125 \mathrm{ps}$ ), although a small percentage undergoes pick-off annihilation, too. The free positron annihilates with an electron in the molecules into two photons ( $\tau-400 \mathrm{ps})$. The "fourth" LT-component is a distribution of exponentially decaying terms, probably caused by secondary ortho-Ps reactions with mainly positive ions in normal, nonpolar liquids, followed by free positron annihilation. As its intensity is low $[15,16]$ and normal-quality LT-measurements were performed the "fourth" component will mainly be disregarded in the discussion of the LT-results reported in this paper.

The AC-spectra consists of the corresponding four components. The para-Ps intrinsic annihilation gives a narrow peak in the AC-spectra of width determined mainly by the zero-point energy of Ps in its bubble state in a liquid. However, free positron and ortho-ps pick-off annihilation result in broad AC-components as the annihilating electrons are bound in the molecules. The "fourth" AC-component is probably mainly of the free positron annihilation shape. At present we cannot separate the shapes of the free positron and pick-off AC-components for hexane.

Let us first discuss the low concentration results. We measured a clear broadening of the broad AC-components on the addition of $\mathrm{C}_{6} \mathrm{~F}_{14}$ to hexane and isooctane, but not if $\mathrm{C}_{6} \mathrm{~F}_{6}$ was added to 


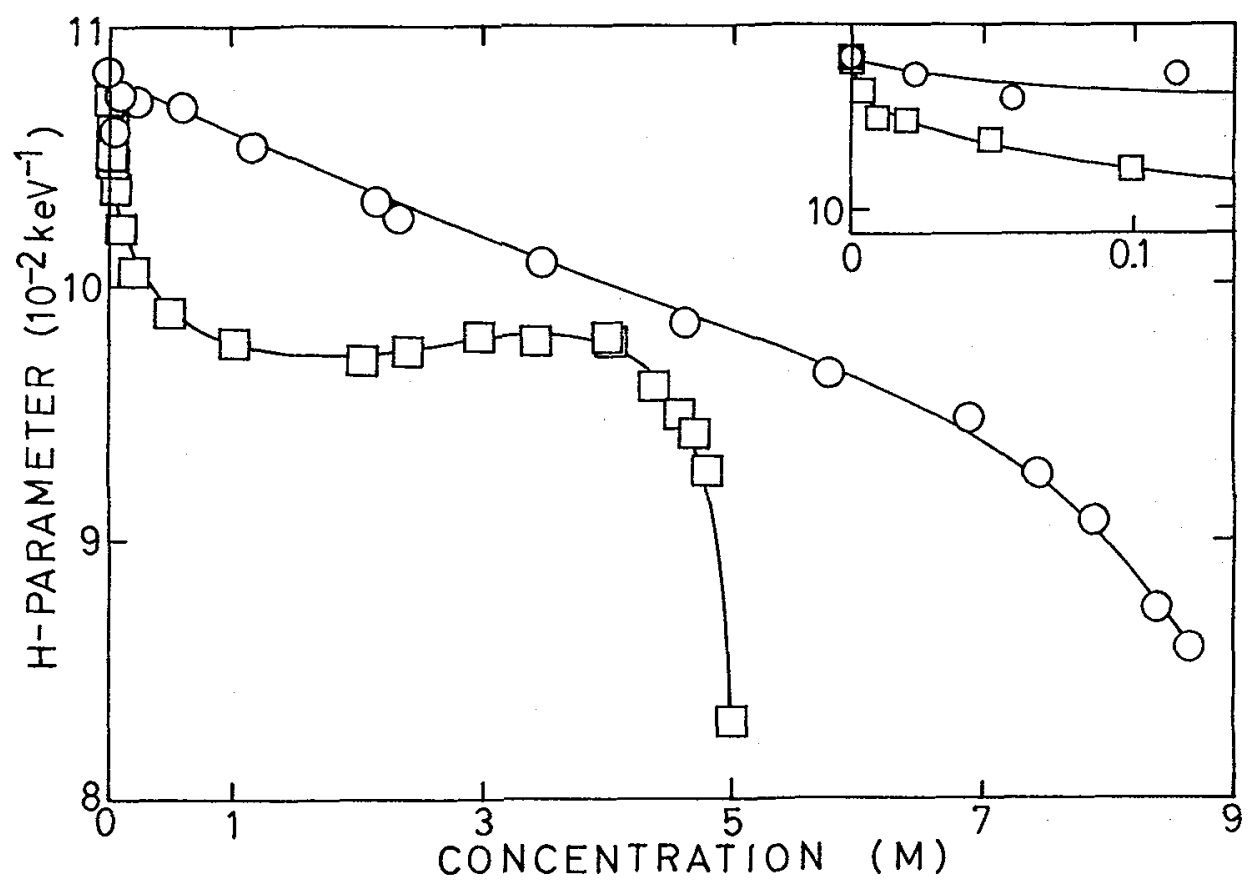

Fig. 5. H-parameter for the broad AC-component versus $\mathrm{C}_{6} \mathrm{~F}_{14}$ concentration in isooctane ( $\square \square \square \square$ ) and $\mathrm{C}_{6} \mathrm{~F}_{6}$ concentration in hexane (OOOO) mixtures. The insert shows the low concentration results. Lines are for guidance only.

hexane. These results were expected if the free positron reacts with $\mathrm{C}_{6} \mathrm{~F}_{14}$ but not with $\mathrm{C}_{6} \mathrm{~F}_{6}$, as discussed in the introduction. However, we expected intuitively to find all the broadening of the broad AC-component at the addition of low concentration of $\mathrm{C}_{6} \mathrm{~F}_{14}$, which is in disagreement with experiments. The fact that the greatest broadening occurred at a very high $\mathrm{C}_{6} \mathrm{~F}_{14}$ concentration was a surprise. It is, therefore, important to discuss in detail all possible, alternative interpretations of the low concentration results.

At first, we must show that the measured broadening of the AC-spectra is well explained by the assumption of a positron reaction with $\mathrm{C}_{6} \mathrm{~F}_{14}$ in hexane and isooctane but not with $\mathrm{C}_{6} \mathrm{~F}_{6}$ in hexane. This is a necessary condition for the validity of that explanation.

Roughly speaking we can assume that the free positron reaction with $\mathrm{C}_{6} \mathrm{~F}_{14}$ occurs in competition with the annihilation, i.e., that the reaction is described by:

$$
d c_{+} d t=-\lambda_{f} c_{+}-k_{f} c_{+} c_{a}
$$

where $\lambda_{f}$ is the positron decay rate $\left(\lambda_{f}=1 / 430 \mathrm{ps}=2.3 \times 10^{9} \mathrm{~s}\right), \mathrm{c}_{+}\left(\mathrm{c}_{\mathrm{a}}\right)$ is the concentration of positrons (solute), and $k_{f}$ is the rate constant. This implies that the amount of reacted positrons, $x$, is given by:

$$
x=k_{f} c_{a} /\left(\lambda_{f}+k_{f} c_{a}\right)
$$


In both liquids half of the broadening at low concentration occurs at about $0.08 \mathrm{M} \mathrm{C}_{6} \mathrm{~F}_{14}$, which by use of (2) gives $k_{f}=3 \times 10^{10} \mathrm{M}^{-1} \mathrm{~s}^{-1}$. Two approximations were used in this derivation. First, we disregarded the nonhomogeneous distribution of the positron spur reactants and their influence of the positron reaction. Second, the concentration is so high that the time-dependent term in the equation for the rate constant may play a role, too (see [11]). On taking into account these approximations the uncertainty is probably about $\pm 10^{10} \mathrm{M}^{-1} \mathrm{~s}^{-1}$. This is a $\mathrm{k}_{\mathrm{f}}$-value expected for a slowly moving, heavy reactant. It is not larger than could reasonably be assumed. Maybe it is even smaller than expected as the positron is fast moving in both liquids. This follows from the existence of the antirecombination effect in both solvents, which indicates that the positron has a higher mobility and diffusion constant than the positive ions $[4,6]$. Hence, we have to inquire into the possibility that other mechanisms can explain the results, too.

The only other mechanism which can be imagined is that the positron in Ps annihilates preferentially with the electrons in the fluorine atoms at low concentrations of $\mathrm{C}_{6} \mathrm{~F}_{14}$ to give the broad component. This would imply that $P s$ reacts with $\mathrm{C}_{6} \mathrm{~F}_{14}$. However, a Ps reaction has always in the past been assumed to take place only if the ortho-Ps lifetime decreases with the concentration of the reactant, while an increase in the ortho-Ps lifetime on the addition of $\mathrm{C}_{6} \mathrm{~F}_{14}$ was measured [10]. Hence, the assumption that the broadening is caused by a reaction of the positron in Ps with the fluorine electrons can be ruled out.

It is well known that a positron annihilation with fluorine in the molecules in a condensed matter results always in a strong broadening of the broad AC-component. Hence, the absence of such broadening in the $\mathrm{C}_{6} \mathrm{~F}_{6}$ / hexane mixture at low $\mathrm{C}_{6} \mathrm{~F}_{6}$ concentration shows clearly that the free positron does not react with $\mathrm{C}_{6} \mathrm{~F}_{6}$ in hexane.

Consequently, we can conclude that the positrons which do not form Ps react with $\mathrm{C}_{6} \mathrm{~F}_{14}$ in hexane and isooctane, but not with $\mathrm{C}_{6} \mathrm{~F}_{6}$ in hexane. This important result is in full agreement with the Ps yield data, as discussed above. We do not know why there is a difference between the two fluorine containing molecules.

The next result to discuss is the measured, strong broadening on the addition of the last percentage of $\mathrm{C}_{6} \mathrm{~F}_{14}$ to hexane and isooctane. Or to put it differently: Why is a strong narrowing of the broad $\mathrm{AC}$ component obtained on the addition of a low concentration of hexane or isooctane to $\mathrm{C}_{6} \mathrm{~F}_{14}$ ? The similar addition of a low concentration of hexane to $\mathrm{C}_{6} \mathrm{~F}_{6}$ results in only a small narrowing. In general, there are only two types of positrons, namely free positrons and positrons in Ps, in the mixtures. Hence, the important question is: Which type of positron gives the strong narrowing? In principle, the possibilities that it is caused by only one of, or both, the two type of positrons must be considered.

Actually, it will be shown that it is difficult to decide which mechanism is responsible by use of the available data. We shall, therefore, discuss each mechanism and its consequences in some detail.

At first, let us assume that the narrowing is caused by a change in the annihilation of the free positron. Almost the only way this can happen seems to be that the positron is, at first, trapped by only one $\mathrm{C}_{6} \mathrm{~F}_{14}$ molecule at low $\mathrm{C}_{6} \mathrm{~F}_{14}$ concentration, resulting in the first decrease in the $\mathrm{H}$ parameter, and that other $C_{6} F_{14}$ molecules prefer not to join the positron- $C_{6} F_{14}$ complex for energy reasons until this cannot any longer be avoided at very high $\mathrm{C}_{6} \mathrm{~F}_{14}$ concentration. Such processes might, in principle, explain the strong broadening at high concentrations. However, it seems to be very unlikely that only one $\mathrm{C}_{6} \mathrm{~F}_{14}$ molecule will trap the positron because the positron is positively charged and the fluorine atoms in the outer parts of $\mathrm{C}_{6} \mathrm{~F}_{14}$ are negatively charged. It is to be expected that more than one $\mathrm{C}_{6} \mathrm{~F}_{14}$ molecule will participate is the bound state of the 
positron and $\mathrm{C}_{6} \mathrm{~F}_{14}$ already at fairly low $\mathrm{C}_{6} \mathrm{~F}_{14}$ concentrations. Furthermore, a shift in the Ps pickoff annihilation from hexane to $\mathrm{C}_{6} F_{14}$ must result in a decrease in $\mathrm{H}$ at some $\mathrm{C}_{6} \mathrm{~F}_{14}$ concentration. The high concentration decrease in $\mathrm{H}$ is very probably the expected pick-off annihilation effect. Hence, we can conclude that the high concentration effect is probably not caused by a change in the free positron annihilation, although we cannot exclude that a small part of the effect is caused by a that effect.

Second, let us assume that the narrowing is caused by a change in the annihilation process of the positron in Ps. Actually, we intuitively would be inclined to assume that annihilation of the positron in Ps causes the high concentration effect, as the decrease in $\mathrm{H}$ at low concentration of $\mathrm{C}_{6} \mathrm{~F}_{14}$ was found to be caused by the free positron annihilating preferentially with fluorine electrons.

One possibility is that the effect is only a Ps yield effect. As shown in figs. 1 and 2, the para Ps yield given by the narrow AC component decreases by $46 \%$, and the ortho Ps yield obtained from the three term fits of the LT-spectra decreases by $32 \%$, on the addition of low concentrations of hexane or isooctane to $\mathrm{C}_{6} \mathrm{~F}_{14}$. Intuitively, one might tend to assume that this change in the Ps yield is the only cause of the high-concentration effects in figs. 3 to 5 . However, only the assumption that the pick-off AC component is much broader than the free positron AC component can explain the effect in that case. Actually, the width of the pick off component seems to be equal to or smaller than that of the free positron component, in the very few cases where the shapes of the AC components have been determined [4,11]. Furthermore, this assumption of only a Ps yield effect is in disagreement with the behaviour of the H-parameter at lower $\mathrm{C}_{6} \mathrm{~F}_{14}$ concentrations. Hence, this mechanism seems not to occur.

Another possibility is that the pick off annihilation of Ps occurs preferentially with the electrons in the hydrocarbons, and that the annihilation with the electrons in the fluorine atoms in $\mathrm{C}_{6} \mathrm{~F}_{14}$ takes place only at the highest $\mathrm{C}_{6} \mathrm{~F}_{14}$ concentrations, where this cannot any longer be avoided. Actually, a decrease in the H-parameter due to a change in the pick-off annihilation of Ps is expected at some $\mathrm{C}_{6} \mathrm{~F}_{14}$ concentrations when the $\mathrm{C}_{6} \mathrm{~F}_{14}$ concentration is increased. Such effect can be explained by the assumption that the energy of Ps in the bubble state in the mixtures is lower if the first layer of the bubble surface is composed of hydrocarbon molecules and higher if some or all of the surface molecules are $\mathrm{C}_{6} \mathrm{~F}_{14}$. Is this a reasonable assumption?

In hexane the radius of the Ps bubble is about $0.6 \mathrm{~nm}$, i.e., the surface area is roughly $4.52 \mathrm{~nm}^{2}$. As the hexane molecules cover approximately $0.4 \times 1.0 \mathrm{~nm}^{2}=0.4 \mathrm{~nm}^{2}$ the number of hexane molecules on the surface is about 11. Hence, Ps interacts with many molecules in the Ps bubble surface, and a small difference in the Ps-molecule interaction energy is, therefore, expected to cause a fairly large change in the total energy of the Ps bubble state. The detailed nature of the Ps-molecule interaction seems to be unknown $[17,18]$. To get an understanding of the problems involved it is appropriate to discuss the Ps bubble state in some detail.

In the simplest approximation the total energy of the Ps bubble state has been given by

$$
E=E_{o}(U, r)+4 \pi r^{2} \gamma+(4 / 3) \pi r^{3} p
$$

$E_{o}(U, r)$ is the zero point energy in the square-well potential of depth $U$ and radius $r$, in which Ps is assumed to be situated.

The second and third terms are the surface energy and pressure times volume terms, respectively. Hence, $\gamma$ is the surface tension and $p$ the pressure. On assuming that the squarewell potential has infinitely high walls we get 


$$
\mathrm{E}_{\mathrm{o}}(\mathrm{U}, \mathrm{r})=\pi^{2} \hbar^{2} /\left(4 \mathrm{~m}_{\mathrm{e}} \mathrm{r}^{2}\right)
$$

where $m_{e}$ is the electron mass and $\hbar$ Planck's constant divided by $2 \pi$. If we further assume that the pv term is very small compared with the other terms, which is normally fulfilled, we get by use of $\mathrm{dE} / \mathrm{dt}=0$ :

and

$$
\mathrm{E}=0.2429 \times \gamma^{1 / 2}
$$

$$
\mathrm{r}=1.242 \times \gamma^{-1 / 4}
$$

where $\gamma$ is in dyne $/ \mathrm{cm}_{2}, \mathrm{E}$ in $\mathrm{eV}$, and $\mathrm{r}$ in $\mathrm{nm}$.

At $20 \mathrm{C}$ for hexane $\gamma=18.40$ dyne $/ \mathrm{cm}$, which gives $\mathrm{E}=1.04 \mathrm{eV}$ and $\mathrm{r}=0.60 \mathrm{~nm}$. We do not know the experimental surface tension of perfluorohexane. However, it can be estimated to be $\gamma=11.9$ dyne/cm by use of an empirical expression ((12-3.6) in [19]), which gives a good accuracy for nonpolar liquids. Hence, $\gamma$ for $\mathrm{C}_{6} \mathrm{~F}_{14}$ is much smaller than that of hexane. For $\mathrm{C}_{6} \mathrm{~F}_{14}$ we get $\mathrm{E}=0.838 \mathrm{eV}$ and $\mathrm{r}=0.67 \mathrm{~nm}$. Consequently, the simplest model of the Ps bubble state predicts that its energy is much lower in $\mathrm{C}_{6} \mathrm{~F}_{14}$ than in hexane. This is the opposite of that indicated by our measurements. Hence, a more detailed model must be used.

One important approximation, used in (3) and (4), is that Ps can be treated as a particle characterized only by its center-of-mass motion. Actually, the positron can penetrate somewhat into the outer electron clouds of the molecules, and it is repelled only by the positive cores of the atoms. For normal molecules which are not electron acceptors, the electron is strongly repelled by the exchange interaction with the outer electrons of the molecules. Consequently, the repulsion between Ps and the molecules is caused by the repulsion of the electron in Ps, and the positron penetrates further into the molecules than the electron in Ps for such molecules. The polarization of Ps in liquids is approximately treated in $[17,18]$, which probably gives the most detailed calculation of the Ps-molecule interaction available in the literature. Apparently, it is very difficult to use the calculated results to estimate the Ps molecule interaction, though. As the Ps bubble state does not contain a heavy center, and since both particles are distributed over a large volume, a reasonably correct treatment of the dispersion and polarization interaction seems to be a very complicated task.

In the simple approximation discussed above the strong exchange repulsion of the electron in Ps, and hence Ps itself, is approximately described by the infinitely high walls. In a higher approximation the dispersion (van-der-Waals) attraction and the attraction due to the polarization of Ps must also be considered. Various examples, in which we still use the approximation of a Ps atom described by its center-of-mass coordinate, will show how this can be done.

A very simple way of taking these effects into account is to add an attractive energy term, $\mathrm{E}_{\mathrm{a}}$, on the right hand side of (3). For example, one can assume that this term is a negative constant which depends on the type of surface molecules. If the constant is numerically larger, by an amount much larger than $\mathrm{kT}$, for hexane than for perfluorohexane the Ps bubble will have a lower energy in the former liquid. Another example is to assume that the attractive term is given by $\mathrm{E}_{\mathrm{a}}=-\mathrm{cr}^{2}$, where $\mathrm{c}$ is a constant, i.e., that $\mathrm{E}_{\mathrm{a}}$ is proportional to the surface of the bubble. This will cause a reduction in energy identical to that given by a corresponding reduction in the surface tension.

A more complicated assumption is to use a potential, $V\left(r_{c}\right)=-c\left(r_{m}-r_{c}\right)^{-6}$, in the Schrødinger equation for the Ps center-of-mass, apart from the square well. Here $r_{m}$ is the distance of the center of the molecules in the first layer of the Ps bubble, and $r_{c}$ that of the Ps center of mass, 
both measured from the center of the bubble. $c$ is a constant. Such potential is the attractive part of the Lennard-Jones potential [19]. The new potential implies that the energy of Ps in the bubble changes away from $\mathrm{E}_{\alpha}$ given by (4). A numerical solution of this Ps eigenvalue problem seems to be possible.

All the three, and many other, similar assumptions of the interaction energy can easily explain the strong increase in the H-parameter (figs. 3-5) on adding of low concentrations of hexane or isooctane to perfluorohexane, if the unknown constants, $c$, dependent on the type of surface molecules, are adjusted to give a lower total Ps bubble energy in the hydrocarbon than in $\mathrm{C}_{6} \mathrm{~F}_{14}$. Hence, it is easy to propose some type of Ps-molecule interaction which can explain the high $\mathrm{C}_{6} \mathrm{~F}_{14}$ concentration effect. However, a reasonably correct interaction is not available, as yet.

The conclusion of the detailed discussion of the measured results is that the decrease in the $\mathrm{H}$ parameter at high $\mathrm{C}_{6} \mathrm{~F}_{14}$ concentration is very probably mainly caused by a lower total energy of the Ps bubble in the hydrocarbons than in $\mathrm{C}_{6} \mathrm{~F}_{14}$. A small contribution to this effect from changes in the free positron annihilation cannot be excluded. This seems to be the first experimental results which clearly indicate that the attractive Ps-molecule interaction must be taken into account in their interpretation. To complete the discussion we shall now discuss some other experimental results which are correlated to those presented. Furthermore. some predictions of the proposed models shall be discussed, too.

Previous experiments have shown that compounds consisting of perfluoronated molecules have larger ortho-Ps pick-off lifetimes, $\tau_{3}$, than expected from their physical properties. A plot of $\tau_{3}$ versus the surface tension, $\gamma$, by Kobayashi [20] shows that $\tau_{3}$ increases monotonously with decreasing $\gamma$. The only liquid for which $\tau_{3}$ is appreciably above that correlation is $\mathrm{C}_{6} \mathrm{~F}_{6}$, which is the only flouronated molecule studied. Also $\tau_{3}$ for $\mathrm{C}_{6} \mathrm{~F}_{14}[10]$ is above that expected from the $\gamma$ value. Additionally, $\tau_{3}$ for liquid $\mathrm{SF}_{6}[21]$ and for liquid and solid teflon [22] are higher than expected. $\mathrm{C}_{6} \mathrm{~F}_{6}$ (electron affinity, $\left.\mathrm{EA}=0.52 \mathrm{eV}\right), \mathrm{C}_{6} \mathrm{~F}_{14}$, and $\mathrm{SF}_{6}(\mathrm{EA}=1.05 \mathrm{eV}$ ) (and probably also teflon) are electron acceptors.

Another important effect is the following. In aliphatic hydrocarbons the positron in Ps is penetrating further into the molecules than the electron in Ps. Hence, the positive charge is closer to the molecules than the negative charge. The positron is prevented from penetrating still further into the molecules by the attraction of the electron and the repulsion by the positive cores of the molecules. The effect of an external magnetic field on Ps in the bubble state in hexane shows that the Ps contact density, $\alpha$, i.e., the density of the Ps electron at the positron, compared to that in vacuum is smaller than one (0.82 [23]), i.e., Ps is swollen. This $\alpha$-value is a consequence of the charge separation in Ps. For Ps in a bubble in hexane with a nitrobenzene molecule on the surface of the bubble $\alpha=0.1-0.2$ [24]. For nitrobenzene $\mathrm{EA}=1.01 \mathrm{eV}$, and in pure nitrobenzene Ps is not formed. This small $\alpha$-value is very probably caused by the electron in Ps being partly transferred to the lowest unoccupied molecular orbital in nitrobenzene, as is normally assumed in charge-transfer complex formation between heavy molecules. Hence, the electron in Ps penetrates much further into nitrobenzene than the positron. We expect the penetration of the positron in Ps into the molecules compared to that of the Ps electron to be determined mainly by the electron affinity of the molecules in the liquids, which probably is strongly correlated to that in vacuum, namely the EA-value. Furthermore, it is expected that the attractive part of the Ps molecule interaction is dependent on the relative penetration of the two particles in Ps.

It is conjectured that the lower Ps bubble state energy in $\mathrm{C}_{6} \mathrm{~F}_{14}$ than in the hydrocarbons is caused mainly by the electron acceptor property of the $\mathrm{C}_{6} \mathrm{~F}_{14}$ liquid being in between that of the hydrocarbon and nitrobenzene liquids. We expect that the penetration of the two particles in Ps into the molecules is roughly equal in $\mathrm{C}_{6} \mathrm{~F}_{14}$, and that the corresponding smaller Ps polarization 
causes the Ps molecule attraction to be lower in $\mathrm{C}_{6} \mathrm{~F}_{14}$. In general, the relative positron penetration into molecules compared to that of the electron in Ps is expected to vary from the aliphatic hydrocarbon case with strongest positron penetration, through intermediate cases (probably similar to $\mathrm{C}_{6} \mathrm{~F}_{14}$ ) of roughly equal penetration, to the extreme cases of molecules of large electron affinity, such as nitrobenzene, in which the electron penetrates so much further into the molecule than the positron that the Ps state has a higher energy than that of a no-Ps state. It is, therefore, conjectured that the Ps contact density being about 0.8 in hydrocarbons (negative EA) is increased to roughly 1.0 - 1.1 for intermediate cases of small and positive electron affinity. At still larger, increasing electron affinity $\alpha$ will decrease again until at high enough EA-value $\alpha$ becomes very small. At last, EA becomes so large that Ps is unstable. Furthermore, it is conjectured that the contact density decreases from about 1.0 (just above or below) to about 0.82 on adding low concentrations of hexane or isooctane to $\mathrm{C}_{6} \mathrm{~F}_{14}$. The available data seem to indicate that other aliphatic, perfluorinated hydrocarbons (and maybe also $\mathrm{C}_{6} \mathrm{~F}_{6}$ ) will give similar effects than $\mathrm{C}_{6} \mathrm{~F}_{14}$. The expectation of $\alpha$ being slightly above 1.0 in the intermediate cases is reasonable if we take into account that, besides being swollen, Ps is also squeezed in the bubble. An $\alpha$-value higher than 1.0 might thus be expected in the special cases where the squeezing is not fully counterbalanced by the swelling [18].

In summary, the obtained results and the given interpretation can be used to predict several new effects to be measured in the future. Additionally, the new data might be used as guides in the formulation of better theories for the attractive part of the Ps molecule interaction. It is an interesting question whether the particular effects discussed here are properties of only the perfluoronated electron acceptors or whether other, low-electron-affinity molecules give roughly the same effects, too.

The Ps yields in figs. 1 and 2 can be explained by the models discussed previously [4,8,9]. As discussed in the introduction, the Ps yield decreases at increasing $\mathrm{C}_{6} \mathrm{~F}_{14}$ concentration at low $\mathrm{C}_{6} \mathrm{~F}_{14}$ concentration because both the positron and the electron get trapped on $\mathrm{C}_{6} \mathrm{~F}_{14}$. Their mobility and diffusion constant become thereby so low that part of the electron-positron pairs, forming Ps in the pure hydrocarbon, cannot form Ps before the positron decays ( $\tau \sim 400 \mathrm{ps}$ ). At $\mathrm{C}_{6} \mathrm{~F}_{14}$ concentration above about $0.5 \mathrm{M}$ one or both of the particles begin to tunnel from one $\mathrm{C}_{6} \mathrm{~F}_{14}$ molecule to another. The Ps formation becomes thereby somewhat faster, and hence, the Ps yield increases again because more pairs can now form Ps before the positron decays. The minimum in the Ps yield at high $\mathrm{C}_{6} \mathrm{~F}_{14}$ concentration is very probably correlated with a strong, not yet measured, decrease in the excess electron mobility at the same concentration. Actually, based on a similar decrease in the Ps yield in $\mathrm{C}_{6} \mathrm{~F}_{6}$ mixtures with hexane, a decrease in the electron mobility on adding hexane to $\mathrm{C}_{6} \mathrm{~F}_{6}$ was predicted $[4,9]$, and later measured for $\mathrm{C}_{6} \mathrm{~F}_{6}$ added to isooctane, benzene, and cyclohexane [25]. The difference between the apparent Ps yield obtained from AC-spectra and that determined by LT-spectra fitted by three terms is probably caused by the missing Ps yield in the "fourth" LT-component, which is mainly included in the free positron LT-component in a three term analysis $[9,16]$.

It is interesting to note that studies of positron attachment to molecules in vacuum $[26,27]$ showed that fluorocarbons $\left(\mathrm{CF}_{4}, \mathrm{C}_{6} \mathrm{~F}_{14}\right.$, and $\left.\mathrm{C}_{6} \mathrm{~F}_{6}\right)$ have a much lower annihilation rate than the analogous hydrocarbons, if measured under identical conditions. On the other hand, the attachment of positrons to molecules in low pressure gases needs not correlate directly to the energy of positron-molecule bound states measured in liquids, as shown by similar electron attachment studies. 


\section{Conclusion}

The results illustrate that, apart from the normally used lifetime spectroscopy, the angular correlation and contact density measurements might be necessary tools to investigate the processes of the positron and Ps in liquids.

Angular correlation spectra due to positron annihilation with electrons bound in fluorine atoms are broader than those of normal liquids. This fact allows a study of the free positron reactions with perfluoronated hydrocarbons added to hydrocarbons. It was shown that the free positron in hexane and isooctane reacts with $\mathrm{C}_{6} \mathrm{~F}_{14}$ with rate constants of $3 \times 10^{10} \mathrm{M}^{-1} \mathrm{~s}^{-1}$, but not with $\mathrm{C}_{6} \mathrm{~F}_{6}$ in hexane. This fact explains why $\mathrm{C}_{6} \mathrm{~F}_{14}$ inhibits, while $\mathrm{C}_{6} \mathrm{~F}_{6}$ increases, the Ps formation at low concentration in hexane.

Furthermore, it was shown that the Ps bubble has a lower energy in pure $\mathrm{C}_{6} \mathrm{~F}_{14}$ than in pure hexane and isooctane. This is probably caused by a difference in the Ps attraction to the surface molecules of the Ps bubble. In liquids with negative electron affinities, e.g., aliphatic hydrocarbons, the positron in Ps penetrates further into the molecules than the electron in Ps. At increasing electron affinity the electron in Ps begins to penetrate further into the molecules until the electron penetration is much larger than that of the positron in Ps at high electron affinity (e.g., in nitrobenzene). $\mathrm{C}_{6} \mathrm{~F}_{14}$ is probably an intermediate case where the penetration of the two particles in Ps is approximately equal. The Ps molecule attraction is, therefore, relatively weaker in $\mathrm{C}_{6} \mathrm{~F}_{14}$ than in the hydrocarbons. This seems to be the cause of the lower Ps-bubble energy in $\mathrm{C}_{6} \mathrm{~F}_{14}$ than in the hydrocarbons. Various effects in the contact density in the liquids and their mixtures were conjectured.

\section{Acknowledgements}

Many interesting discussions with M. Eldrup and the technical support of N. J. Pedersen throughout this work are acknowledged. 


\section{References}

[1] L. Dorikens-Vanpraet, M. Dorikens and D. Segers, eds., Positron Annihilation, Proceedings of the Eighth Conference of Positron Annihilation, Gent, Belgium, 1988 (World Scientific, Singapore).

[2] Zs. Kajcsos and Cs. Szeles, eds., Positron Annihilation, Proceedings of the Ninth Conference of Positron Annihilation, Szombathely, Hungary, 1991, Materials Sci. Forum 105-110 (1992) 12070 (Trans Tech. Publ., Aedermannsdorf, Switzerland, 1992).

[3] D. M. Schrader and Y. C. Jean, eds., Positron and Positronium Chemistry (Elsevier, Amsterdam, 1988).

[4] O. E. Mogensen, Positron Annihilation in Chemical Physics (Springer, Heidelberg, to be published).

[5] J. W. T. Spinks and R. J. Wood, An Introduction to Radiation Chemistry (J. Wiley, New York, 1990).

[6] J. M. Warman, in The Study of Fast Processes and Transient Species by Electron Pulse Radiolysis, ed. by J. E. Baxendale and F. Busi, (Reidel, Dordrecht, 1982) p. 433.

[7] B. Levay and O. E. Mogensen, Chem. Phys. $\underline{53}$ (1980) 131.

[8] G. Wikander, O. E. Mogensen and N. J. Pedersen, Chem. Phys. 77 (1983) 159.

[9] O. E. Mogensen and F. M. Jacobsen, Chem. Phys. $\underline{73}$ (1982) 223.

[10] G. Wikander, Chem. Phys. Lett. $\underline{80}$ (1981) 361.

[11] O. E. Mogensen and N. J. Pedersen, Radiat. Phys. Chem. 28 (1986) 33.

[12] O. E. Mogensen, N. J. Pedersen and F. M. Jacobsen, in: P. C. Jain, R. M. Singru and K. P. Gopinathan, eds., Positron Annihilation (World Scientific, Singapore, 1985) p. 205.

[13] P. Kirkegaard, M. Eldrup, O. E. Mogensen and N. J. Pedersen, Comp. Phys. Comm. 23 (1981) 307.

[14] P. Kirkegaard, N. J. Pedersen and M. Eldrup, PATFIT-88, Risø-M-2740 (1989).

[15] F. M. Jacobsen, O. E. Mogensen and N. J. Pedersen, in [1] p. 651.

[16] T. Hirade and O. E. Mogensen, Chem. Phys., in press.

[17] J. Chen and M. J. Stott, in [2] p. 1513.

[18] T. McMullen and M. J. Stott, Can. J. Phys. 61 (1983) 504.

[19] R. C. Reid, J. M. Prausnitz and B. C. Poling, The Properties of Gases and Liquids (McGrawHill, New York, 1988) 4th ed..

[20] Y. Kobayashi, J. Chem. Soc. Faraday Trans. 87 (1991) 3641.

[21] F. M. Jacobsen, M. Eldrup and O. E. Mogensen, Chem. Phys. 50 (1980) 393.

[22] L. Breitenhuber, P. Kindl, H. Sorman and W. Puff, Proc. Int. Conf. on Amorphous Systems Investigated by Nuclear Methods, Balatonfüred, Hungary, 1 (1981) 327.

[23] D. A. Diehl and D. M. Schrader, J. Chem. Phys. 94 (1991) 289.

[24] O. E. Mogensen, Chem. Phys. Lett. 163 (1989) 145.

[25] C. A. M. van den Ende, L. Nyikos, J. M. Warman and A. Hummel, Radiat. Phys. Chem. 19 (1975) 297.

[26] T. J. Murphy and C. M. Surko, Phys. Rev. Lett. 67, (1991) 2954.

[27] S. Tang, M. D. Tinkle, R. D. Greaves and C. M. Surko, Phys. Rev. Lett. $\underline{68}$ (1992) 3793. 